\title{
Stereocomplexed Poly(lactic acid) - Poly(ethylene glycol) Nanoparticles with Dual-Emissive Boron Dyes for Tumor Accumulation
}

\author{
Farrell R. Kersey, ${ }^{\dagger}$ Guoqing Zhang, ${ }^{\dagger}{ }^{\text {Gregory M. Palmer, }}{ }^{\ddagger}$ Mark W. Dewhirst, ${ }^{\ddagger}$ and Cassandra L. Fraser*, \\ ${ }^{\dagger}$ Department of Chemistry, University of Virginia, McCormick Road, Charlottesville, Virginia 22904 and ${ }^{\ddagger}$ Department of Radiation Oncology, Duke University Medical \\ Center, Durham, North Carolina 27710
}

L ong-circulating "stealth" nanoparticles (NPs) play an important role in biomaterials and medical fields given their role as efficient tumor-uptake vehicles. Amphiphilic block copolymers are often used to create NP assemblies of this nature. ${ }^{1,2}$ The hydrophobic blocks aggregate to form a core structure, where molecules such as drugs may be contained for delivery, ${ }^{3-6}$ and the hydrophilic segments act as a corona to make the NPs watersoluble. These hydrophilic segments can also aid in the passive targeting of tumors, where they are taken up by the enhanced permeability and retention (EPR) effect, ${ }^{7,8}$ or possess terminal ligands such as biotin ${ }^{9-11}$ or antibodies ${ }^{12}$ for active targeting. Several hydrophilic and hydrophobic polymer segments have been previously utilized; however, poly(ethylene glycol) (PEG) and poly(lactic acid) (PLA) are the most common, respectively. ${ }^{4,5,13-20}$ In addition to $D, L-P L A$ alone as the hydrophobic segment of NPs, stereocomplexation of D- and L-PLA has been utilized to create structures with greater stability and longer shelf lives. . $^{5} 16,21-24$

It is also desirable to add optical imaging and sensing functionality to these delivery systems for cellular and in vivo uses. Imaging agents such as fluorescent difluoroboron-based dyes based on $\mathrm{BO}$ DIPY ${ }^{25}$ and $\beta$-diketonate derivatives, ${ }^{26}$ and heavy metal reagents, ${ }^{27,28}$ have been previously utilized for such purposes. Imaging agents of this nature are often incorporated into a polymeric matrix to create blends that enhance dye stability and reduce toxicity in biological systems. However, the use of dye-polymer conjugates minimizes dye www.acsnano.org

ABSTRACT Responsive biomaterials play important roles in imaging, diagnostics, and therapeutics. Polymeric nanoparticles (NPs) containing hydrophobic and hydrophilic segments are one class of biomaterial utilized for these purposes. The incorporation of luminescent molecules into NPs adds optical imaging and sensing capability to these vectors. Here we report on the synthesis of dual-emissive, pegylated NPs with "stealth"-like properties, delivered intravenously (IV), for the study of tumor accumulation. The NPs were created by means of stereocomplexation using a methoxy-terminated polyethylene glycol and poly(D-lactide) (mPEG-PDLA) block copolymer combined with iodide-substituted difluoroboron dibenzoylmethane-poly(L-lactide) ( $\left.\mathrm{BF}_{2} \mathrm{dbm}(\mathrm{I}) \mathrm{PLLA}\right)$. Boron nanoparticles (BNPs) were fabricated in two different solvent compositions to study the effects on BNP size distribution. The physical and photoluminescent properties of the BNPs were studied in vitro over time to determine stability. Finally, preliminary in vivo results show that stereocomplexed BNPs injected IV are taken up by tumors, an important prerequisite to their use as hypoxia imaging agents in preclinical studies.

KEYWORDS: Boron dye - fluorescence - phosphorescence - nanoparticles · poly(lactic acid) (PLA) stereocomplexes · poly(ethylene glycol) (PEG) · enhanced permeation and retention (EPR) effect

leaching along with heterogeneities associated with dye-polymer blends. ${ }^{29}$ Previous studies have also shown that such materials have the potential to act as ratiometric sensing agents, often utilizing heavy metals $^{30-33}$ or possessing multiple components. ${ }^{34-36}$ Examples of these materials include fluorescent imaging compounds combined with an oxygen-sensing phosphorescent component, ${ }^{36}$ as well as ratiometric sensors which exhibit only phosphorescence. ${ }^{37,38}$

We have previously reported on the fabrication of difluoroboron dibenzoylmethane poly(lactic acid) $\left(\mathrm{BF}_{2} \mathrm{dbmPLA}\right)$ powders and films, ${ }^{39,40}$ as well as BNPs which exhibited both intense blue fluorescence and green room-temperature phosphorescence (RTP) in aqueous suspension. ${ }^{41,42}$ Additionally, BNPs of the iodide analogue $\mathrm{BF}_{2} \mathrm{dbm}(\mathrm{I}) \mathrm{PLA}$ were prepared that showed more practical
*Address correspondence to fraser@virginia.edu.

Received for review December 21, 2009 and accepted July 19, 2010.

Published online August 12, 2010. $10.1021 / \mathrm{nn} 901873 \mathrm{t}$

() 2010 American Chemical Society 
features for oxygen sensing in vivo, making them useful as ratiometric tumor hypoxia imaging agents for preclinical studies. ${ }^{43}$ Though valuable for direct tissue injection, NPs such as those previously reported by our group are not suitable for intravenous delivery due to their lack of a coronal segment such as PEG, which is necessary to impart stealth properties and long circulation as well as facilitate tumor uptake by the EPR effect. Thus, systems containing a boron dye, PLA, and PEG were targeted. Given that the boron dye is not stable to many basic and nucleophilic reagents that are typical for PEG-PLA coupling reactions, stereocomplexation of PLA segments was explored as an alternative, due to synthetic challenges involved in creating an mPEG-PLA-dye covalent system. Stereocomplexation is a simple, convenient, and modular approach to creating pegylated PLA NPs and allows us to test whether tumor uptake is possible with this third generation BNP system. It has been previously observed that sterecomplexation of PDLA and PLLA in MPEG-PLA NPs leads to smaller NP sizes when compared to PDLLA, PDLA, and PLLA NPs. ${ }^{21}$ These smaller sizes can be beneficial for uptake and stealth-like properties; however, stereocomplexed NPs could degrade faster than NPs created from a covalent system both in vitro and in vivo. Here we test these hypotheses by fabricating BNPs from two solvent systems, investigating their properties and stability in vitro and their accumulation and imaging capability in vivo in a mouse dorsal window chamber model.

\section{RESULTS AND DISCUSSION}

lodide-substituted difluoroboron dibenzoylmethane-poly(L-lactide) $\left(\mathrm{BF}_{2} \mathrm{dbm}(\mathrm{I}) \mathrm{PLLA}, 1\right)^{43}$ and methoxy-terminated poly(ethylene glycol)- $b$ poly(D-lactide) (mPEG-PDLA, 2) ${ }^{15}$ (Figure 1) were synthesized by ring-opening polymerization using the catalyst $\mathrm{Sn}(\mathrm{oct})_{2}$ as previously described. Nanoparticle fabrication was performed using the nanoprecipitation method where both $\mathbf{1}$ and $\mathbf{2}$ were dissolved in a watermiscible solvent or solvent mixture (e.g., DMF, THF), followed by dropwise addition of the polymer solution to water, leading to NP formation, as shown in Figure 1. ${ }^{15,41,44}$ For these studies, DMF and DMF/THF (10:1 v/v) were chosen as the solvent phases. DMF is commonly chosen as the solvent phase due to its propensity to create NPs with small $(<100 \mathrm{~nm}$ ) diameters; however, the DMF/THF binary solvent mixture was also tested due to the poor solubility of PEG in DMF. The organic phase was subsequently removed from the aqueous medium by means of dialysis. Following fabrication, the size, optical properties, and dye/polymer stability of the stereocomplexed BNPs were studied.

Nanoparticle sizes were determined using dynamic light scattering (DLS). DLS measurements showed that when the DMF/THF solvent phase was used, the resulting BNPs possessed a hydrodynamic diameter $\left(D_{\mathrm{h}}\right)$ of $120 \pm 20 \mathrm{~nm}$, with a polydispersity $(\mathrm{Pd})$ of $0.28 \pm 0.05$.<smiles></smiles>

1<smiles>COC(C)COC(C)COC(=O)C(C)O</smiles>

2

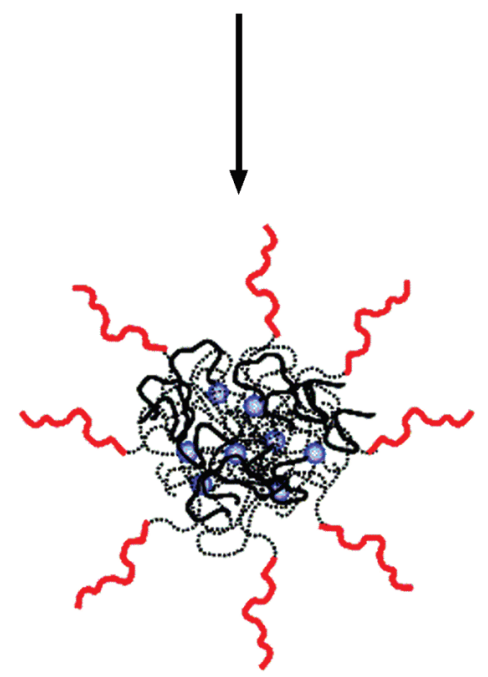

Figure 1. Chemical structures of $\mathrm{BF}_{2} \mathrm{dbm}(\mathrm{I}) \mathrm{PLLA}(1)$ and mPEG-b-PDLA (2) used to prepare pegylated BNPs, shown schematically. The NP corona is composed of MPEG chains (red lines), while the core contains PLLA (dashed lines) and PDLA (black lines). $\mathrm{BF}_{2} \mathrm{dbm}(\mathrm{I})$ dye molecules are shown as blue spheres within the stereocomplex core.

However, when DMF was used as the solvent phase, both $D_{\mathrm{h}}$ and $\mathrm{Pd}$ of the BNPs were reduced to $83 \pm 7 \mathrm{~nm}$ and $0.17 \pm 0.03$, respectively. These findings correlate well with previous studies, as the use of DMF as the solvent phase has been shown to result in NPs with diameters ranging from 40 to $80 \mathrm{~nm}$, whereas the use of THF gives rise to larger NPs $(\sim 240 \mathrm{~nm}) .{ }^{17}$

After fabrication, the BNPs were characterized by UV-vis and ${ }^{1} \mathrm{H}$ NMR spectroscopy and gel permeation chromatography (GPC). This was achieved by first freeze-drying a sample of the aqueous BNP suspension immediately after fabrication and redissolving the sample in an organic solvent suitable for the method of characterization. The extinction coefficient $\epsilon$ for postfabricated freeze-dried NPs $\left(3.9 \times 10^{4} \mathrm{M}^{-1} \mathrm{~cm}^{-1}\right)$ was found to be similar to that of the starting material 1 (4.2 $\left.\times 10^{4} \mathrm{M}^{-1} \mathrm{~cm}^{-1}\right)$ in $\mathrm{CH}_{2} \mathrm{Cl}_{2}$. Additionally, $\lambda_{\max }$ was found to be $405 \mathrm{~nm}$ before and after fabrication for both in $\mathrm{CH}_{2} \mathrm{Cl}_{2}$. These results indicate that virtually no damage is imparted to the boron dye component of $\mathbf{1}$ during processing. ${ }^{1} \mathrm{H}$ NMR spectra of the freeze-dried BNPs showed the complete removal of the organic solvent phase. Because GPC RI traces for $\mathrm{BF}_{2} \mathrm{dbm}(\mathrm{I}) \mathrm{PLLA}$ and 

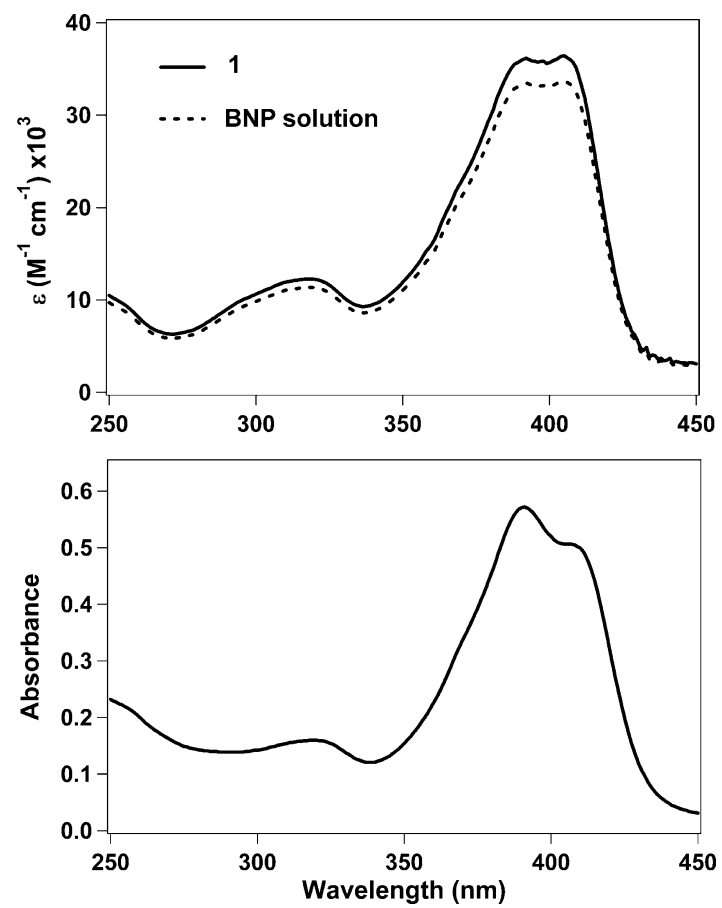

Figure 2. UV - vis absorption spectra for $1(\sim 3.7 \mu \mathrm{M})$ and freeze-dried nanoparticles (BNP, $\sim 3.4 \mu \mathrm{M}$ ) dissolved in $\mathrm{CH}_{2} \mathrm{Cl}_{2}$ (top) and for the stereocomplexed nanoparticles in aqueous suspension ( $\sim 0.13 \mathrm{mg} / \mathrm{mL}$; bottom).

PDLA-PEG in the stereoblock sample overlap, direct comparison of the stereoblock mixture with its individual polymer constituents is not possible. However, GPC with UV detection, which only records $\mathrm{BF}_{2} \mathrm{dbm}(\mathrm{I}) \mathrm{PLLA}$ elution, indicates an $M_{\mathrm{n}}=4.9 \mathrm{kDa}$ versus $M_{\mathrm{n}}=5.7 \mathrm{kDa}$ for the $\mathrm{BF}_{2} \mathrm{dbm}(\mathrm{I})$ PLLA starting material. It is important to note, however, that the presence of PEG-PDLA in the sample mixture could influence the GPC elution volume for $\mathrm{BF}_{2} \mathrm{dbm}$ (I)PLLA.

The optical properties of the aqueous BNP suspensions were studied using UV-vis and fluorescence spectroscopy. As shown in Figure 2, the UV-vis spectrum of the freshly prepared, freeze-dried BNPs in $\mathrm{CH}_{2} \mathrm{Cl}_{2}$ exhibit an absorption maximum at $405 \mathrm{~nm}$ with a smaller peak at $392 \mathrm{~nm}$, identical to the spectrum for the starting material 1. In contrast, the spectrum of the aqueous BNP suspension shows a shift in these two peaks, with an absorption maximum at $391 \mathrm{~nm}$ and a minor shoulder peak at $406 \mathrm{~nm}$. When the fluorescence spectra were measured in $\mathrm{CH}_{2} \mathrm{Cl}_{2}$, solutions of freezedried BNPs displayed a maximum emission seen at $\lambda_{\mathrm{em}}$ $=438 \mathrm{~nm}$. In contrast, BNPs in aqueous suspensions displayed a red-shifted fluorescence at $\lambda_{\mathrm{em}}=454 \mathrm{~nm}$. This red shift in fluorescence has been observed previously and may indicate dye-dye interactions within individual BNPs. ${ }^{41}$

When BNPs were fabricated using the DMF/THF solvent phase, both fluorescence and phosphorescence lifetimes for the aqueous BNP suspensions could be fit to a triple exponential decay, where the percentweighted lifetimes were found to be $0.46 \mathrm{~ns}$ and 4.6
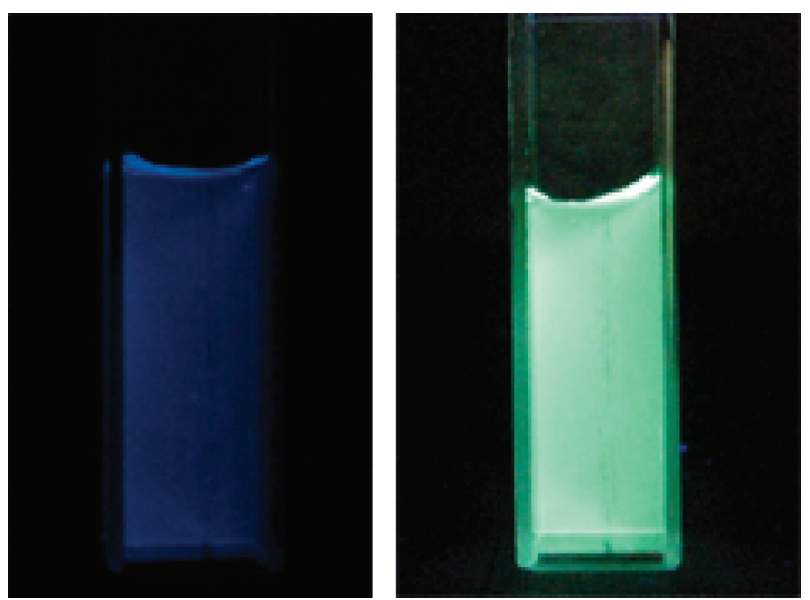

Figure 3. Images showing blue fluorescence (left) and green-yellow phosphorescence (right) of the stereocomplexed BNP suspensions in aqueous medium. Fluorescence is measured under air, while phosphorescence is measured under a nitrogen atmosphere $\left(\lambda_{\mathrm{ex}}=365 \mathrm{~nm}\right)$.

ms for fluorescence and phosphorescence, respectively. Similarly, when fabricated from DMF, fluorescence and phosphorescence lifetimes were found to be $0.50 \mathrm{~ns}$ and $4.3 \mathrm{~ms}$, respectively. These findings are consistent with lifetimes seen for aqueous BNP suspensions prepared from $\mathbf{1}^{43}$ and indicate that processing conditions did not significantly affect luminescence lifetimes.

As was previously reported for NP suspensions of $\mathrm{BF}_{2} \mathrm{dbmPLA}^{41}$ and the iodide-substituted analogue, ${ }^{43}$ long-lived room-temperature phosphorescence (RTP) ${ }^{45}$ and high sensitivity to oxygen quenching are also observed for the stereocomplexed BNPs in this study. Under atmospheric conditions aqueous BNP suspensions exhibit a blue emission when excited at $365 \mathrm{~nm}$ (Figure 3). However, when purged with nitrogen for $20 \mathrm{~min}$, the BNP suspensions exhibited a strong, green-yellow RTP at $\lambda_{\mathrm{em}}=530 \mathrm{~nm}$, with a delayed fluorescence seen at $\lambda_{\mathrm{em}} \approx 450 \mathrm{~nm}$ (Figures 3 and 4). In order to determine the sensitivity of the BNPs to oxygen quenching, the aqueous suspensions were purged with various $\mathrm{O}_{2} / \mathrm{N}_{2}$ gas mixtures with $\mathrm{O}_{2}$ concentrations ranging from $0 \%$ (pure $\mathrm{N}_{2}$ ) to $21 \%$ (ambient conditions), and the fluorescence/phosphorescence intensity ratios (F/P) were recorded. The emission spectra, normalized against fluorescence maxima, $\lambda_{F}$, show how RTP decreases as a function of increasing $\mathrm{O}_{2}$ concentration (Figure 5). As shown in Figure 6 a plot of $\mathbf{F} / \mathbf{P}$ vs $\mathrm{O}_{2}$ concentration exhibits a linear trend between $0 \%$ and $\sim 3 \%$ $\mathrm{O}_{2}$, similar to that for $\mathrm{BF}_{2} \mathrm{dbm}(\mathrm{I}) \mathrm{PLA}$ NPs. ${ }^{43}$ This extended linear trend makes this system especially wellsuited for hypoxic tissue imaging in biological systems, where $\mathrm{O}_{2}$ concentrations typically range from $0.1 \%$ to $1 \%$.

In order to determine the shelf life of the sterecomplexed BNPs, their stability was monitored over a period of several months using DLS, GPC, ${ }^{1} \mathrm{H} N M R, U V-$ vis, and fluorescence spectroscopy. After approximately 4 


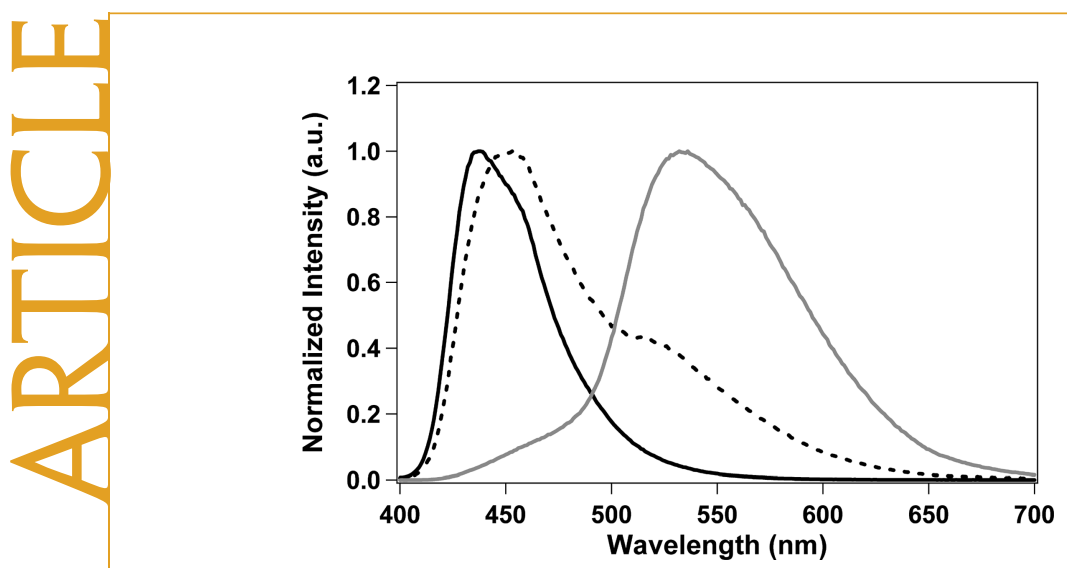

Figure 4. Normalized emission spectra for freeze-dried BNPs in $\mathrm{CH}_{2} \mathrm{Cl}_{2}$ solution (black) and as an aqueous BNP suspension (dashed: fluorescence; gray: phosphorescence). The phosphorescence spectrum was collected under $\mathrm{N}_{2}$. The fluorescence $\boldsymbol{\lambda}_{\mathrm{ex}}$ was $369 \mathrm{~nm}$, while the phosphorescence $\lambda_{\mathrm{ex}}$ was $391 \mathrm{~nm}$.

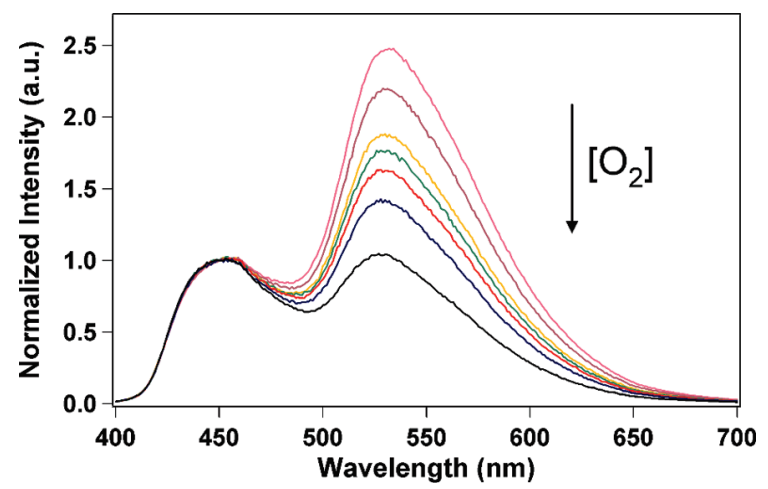

Figure 5. Emission spectra of aqueous BNP solution under increasing oxygen levels (indicated by the arrow, $0-2.6 \%$ in $\mathrm{N}_{2}$ ) normalized to the fluorescence $\lambda_{\mathrm{F}}$.

months the sizes and polydispersities of BNPs fabricated using the DMF/THF mixture, as measured by DLS, remained virtually unchanged $\left(D_{\mathrm{h}}=136 \pm 25 \mathrm{~nm} ; \mathrm{Pd}\right.$ $=0.26 \pm 0.05)$. Though the sizes of the NPs seem to remain consistent over time, some aggregation and sedimentation are visible in the aqueous suspensions after this period of time. GPC analysis shows that the $\mathrm{BF}_{2} \mathrm{dbm}(\mathrm{l}) \mathrm{PLLA}$ constituent of the NPs exhibits good stability over several weeks, as no changes in molecular weight or peaks corresponding to smaller molecular weights are observed. Aqueous BNP suspensions that were freeze-dried after a period of approximately 2 months were noticeably more difficult to dissolve in THF than freeze-dried samples that were less than 1 month old. Attempts to dissolve these older samples were not improved by means of sonication or heating. Additionally, a small shoulder at higher molecular weights can be seen in the UV signal of GPC traces of the older NP samples (see Supporting Information). This shoulder is most likely the result of BNP aggregation or possibly due to polymer cross-linking via peroxidation induced by $\mathrm{PEG}^{46}$ or photochemical reactions with the $\mathrm{BF}_{2} \mathrm{dbm}(\mathrm{I})$ dye. ${ }^{47,48}$ It may be the case that freezedrying of these NPs leads to crystallization of the PEG segments, which makes them difficult to redissolve in

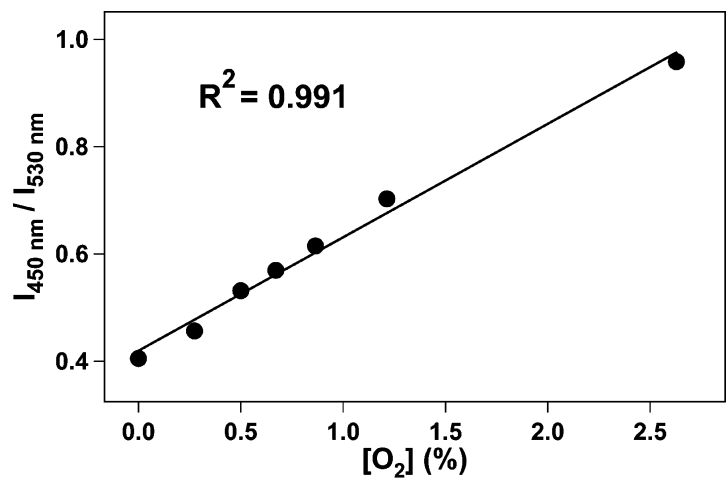

Figure 6. Linear relationship between fluorescence/phosphorescence (F/P) intensity ratio at two fixed wavelengths $(450$ and $530 \mathrm{~nm})$ and oxygen concentration.

certain solvents such as water or THF, as was the case here. This is not an unlikely scenario, as aqueous solutions of proteins and suspensions of pegylated NPs are typically mixed with a lyoprotectant prior to freezedrying to prevent aggregation and allow for redistribution in water. ${ }^{15,49,50}$

Over a period of several weeks, ${ }^{1} \mathrm{H}$ NMR spectra of freeze-dried NPs in $\mathrm{CDCl}_{3}$ exhibit signs of dye degradation. Aryl and enol peaks that correlate with the $\mathrm{dbm}(\mathrm{I}) \mathrm{PLLA}$ macroligand were observed, suggesting hydrolysis of $\mathrm{BF}_{2}$ from the diketonate binding site. The boronated dye remaining over time (10 days: $\sim 75 \% ; 42$ days: $\sim 59 \%$; 81 days: $\sim 34 \%$ ) appears to follow a faster rate of degradation in pegylated stereocomplexes than that for $\mathrm{BF}_{2} \mathrm{dbmPLA} N$ Ps previously fabricated, which showed hydrolysis products present after approximately 11 weeks. ${ }^{41}$ Consistent with the NMR findings, dye degradation is also observed as a decrease in both the $\mathbf{P} / \mathbf{F}$ ratio of $\mathrm{N}_{2}$-purged aqueous BNP samples and the calculated UV-vis extinction coefficient $\epsilon\left(\mathrm{M}^{-1}\right.$ $\mathrm{cm}^{-1}$ ) at $405 \mathrm{~nm}$ over time ( 17 days: $3.0 \times 10^{4} ; 39$ days: $2.4 \times 10^{4} ; 81$ days: $7.5 \times 10^{3}$ ), and the results found by all three methods follow similar trends in rate of degradation (Figure 7). Although some data point scattering is observed in the UV-vis data set, this is most likely a result of the small sample masses $(\sim 0.5-2 \mathrm{mg})$ used to create the $\mathrm{CH}_{2} \mathrm{Cl}_{2}$ solutions.

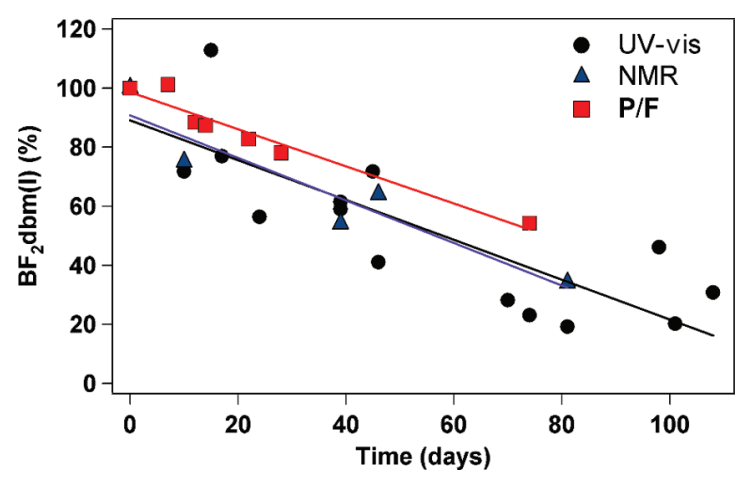

Figure 7. Plot of $\mathrm{BF}_{2} \mathrm{dbm}(\mathrm{I})$ dye degradation over time as measured by UV-vis, 'H NMR, and fluorescence spectroscopy. 
a.

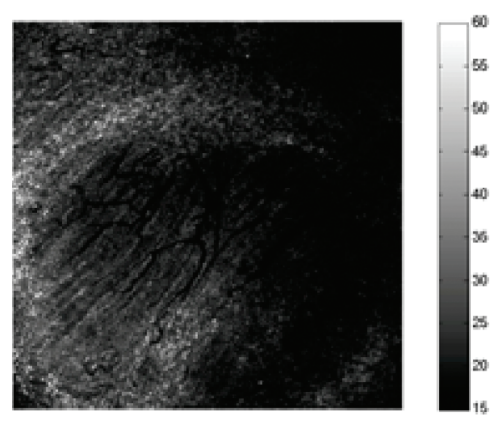

b.

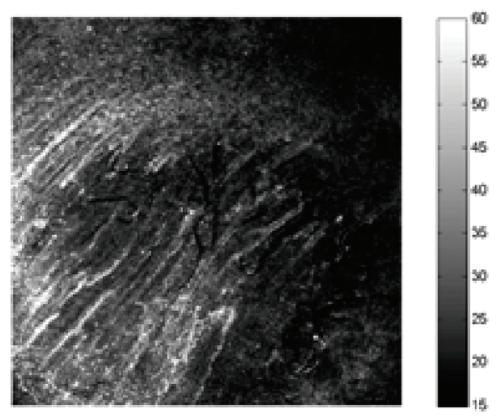

c.

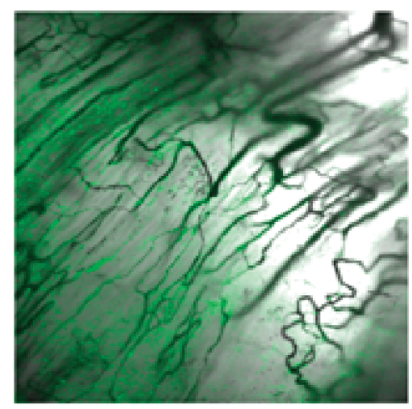

d.

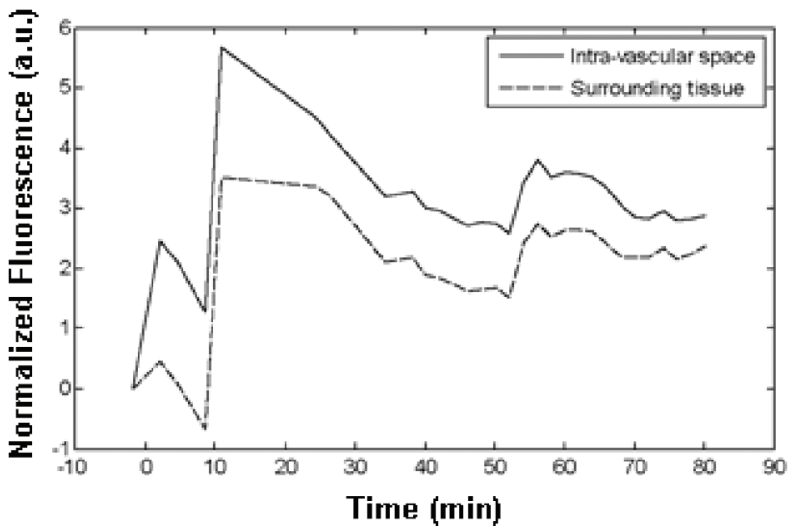

Figure 8. Fluorescence images showing BNP uptake (a) prior to injection and (b) 10 min after injection. (c) Overlay of fluorescence with transmission image. (d) Plot of fluorescence emission over time for vascular and surrounding tissue. All images are $1.55 \times 1.55 \mathrm{~mm}$.

Fluorescence and phosphorescence spectra of the aqueous BNP suspensions show that the relative phosphorescence intensity of $\mathrm{N}_{2}$-purged samples gradually decreases over a period of several weeks, as shown in the $\mathbf{P} / \mathbf{F}$ intensity ratio-versus-time data set in Figure 7 (see also Supporting Information). In addition to the decrease in phosphorescence, a blue shift is observed in the delayed fluorescence $\lambda_{D F}$ (day 0: $\lambda_{D F}=456 \mathrm{~nm}$; 11 weeks: $\lambda_{D F}=448 \mathrm{~nm}$ ). This blue shift may be attributed to the degradation of $\mathrm{BF}_{2} \mathrm{dbm}(\mathrm{I})$ fluorophores. With fewer fluorophores per nanoparticle, the interactions among them may be reduced and, therefore, a larger gap of singlet-triplet energy in the excited state may be expected. This larger gap, based on previous assumptions, can decrease the intersystem crossing rate from singlet to triplet states, resulting in reduced relative RTP intensity. However, another possibility to consider is that the degraded $\mathrm{BF}_{2} \mathrm{dbm}(\mathrm{I})$ product, $\mathrm{dbm}(\mathrm{I})$, is a diketone with the potential to quench RTP from remaining $\mathrm{BF}_{2} \mathrm{dbm}(\mathrm{I})$ fluorophores given its more significant $n-\pi^{*}$ transition nature. ${ }^{43}$ Even though the dye degrades over a period of several months, the bright green-yellow RTP is still visible under a nitrogen atmosphere after this time and the partially degraded material may still serve as a viable ratiometric sensor after calibration.
In vivo experiments using mouse models were performed to examine tumor accumulation of the pegylated BNPs. Figure 8 a shows the fluorescence image using 420-495 $\mathrm{nm}$ band-pass emission prior to injection, and Figure $8 \mathrm{~b}$ shows the fluorescence image at $10 \mathrm{~min}$ postinjection, which was found to be the approximate time of peak uptake. Punctate regions of bright fluorescence appear to be clustered proximal to the blood vessels, which can be seen in the overlay with the transmission image (Figure 8c). The time course is plotted for the vascular and surrounding tissues which shows rapid uptake, peaking around $10 \mathrm{~min}$, with a slow falloff from there (Figure 8d). With this method we cannot distinguish cellular uptake from accumulation in the extracellular extravascular space, so the intensity is a reflection of the total accumulation. The phosphorescence channel (496-623 nm; not shown) did not show a significant increase over baseline, possibly due to high tissue autofluorescence or the long lifetime of the $\mathrm{BF}_{2} \mathrm{dbm}(\mathrm{l})$ dye relative to the scanning speed of the present detection system. Note that the confocal microscopy system used here to investigate BNP vascular localization is different than the wide-field fluorescence microscope previously used to detect phosphorescence for the purposes of ratiometric sensing using nonpegylated BNPs injected directly into the tissue. ${ }^{43}$ Future studies will in- 
volve alternative imaging systems optimized for hypoxia imaging with dual emissive pegylated BNPs.

\section{CONCLUSION}

In summary, dual-emissive pegylated boron nanoparticles were fabricated by means of stereocomplexation between $\mathrm{BF}_{2} \mathrm{dbm}(\mathrm{I}) \mathrm{PLLA}$ and $\mathrm{mPEG}-\mathrm{PDLA}$. Findings show that BNP sizes can be controlled using individual (DMF) or multicomponent (DMF/THF) solvent phases and that the use of DMF alone leads to smaller nanoparticles, consistent with prior reports. ${ }^{41,43}$ Additionally, data suggest that the dye component of the NP is not damaged during the fabrication process. The size properties of the BNPs were found to remain relatively stable over a period of several months; however, aggregation is visibly present in the suspensions and evidenced by DLS and GPC after several weeks. The optical properties of the aqueous pegylated BNP suspensions were found to be well matched to those of nonpegylated BNP suspensions fabricated from starting material 1, displaying both strong fluorescence and long-lived RTP. Similarly, the pegylated BNPs were found to be sensitive to oxygen levels, exhibiting a linear trend in the $\mathbf{F} / \mathbf{P}$ intensity ratio up to an $\mathrm{O}_{2}$ concentration of $\sim 3 \%$, making these NPs suitable for use as hypoxia imaging agents. Degradation of the dye component is readily observed by spectroscopic methods, pointing to the possibile hydrolysis of the $\mathrm{BF}_{2}$ species; however, phosphorescence is still observed when aqueous suspensions of BNPs are subjected to nitrogen purging. In addition to the physical and optical property studies, in vivo studies showed BNP uptake by tumor tissue, demonstrating their stealth-like nature. Future studies will involve optimizing and monitoring BNP uptake by tumors via the EPR effect after intravenous injection, with the aim of using these pegylated BNPs in vivo to record oxygen levels within tumor regions by means of ratiometric sensing based on the dualemissive nature of the boron dyes. ${ }^{43}$ The effects of polymer block lengths on dye and particle stability and the relationship between nanoparticle size and tumor uptake and hypoxia imaging are also subjects of further study.

\section{METHODS}

Materials and Instrumentation. (3S)-cis-3,6-Dimethyl-1,4-dioxane2,5-dione (L-lactide, Aldrich) was recrystallized twice from ethyl acetate and stored under nitrogen. D-Lactide (99.5\%) was a generous gift provided by Purac. Polyethylene glycol 2000 monomethyl ether (mPEG, Fluka) was azeotropically distilled from toluene, freeze-dried from benzene, and stored under nitrogen. 1 and $\mathbf{2}$ were synthesized as previously described. Syringe filters (13 mm, disposable filter device, $0.2 \mu \mathrm{m}$ nylon filter membrane) were obtained from Whatman. All other reagents and solvents were used as received without further purification. Polymer molecular weights were determined by gel permeation chromatography (tetrahydrofuran, $20^{\circ} \mathrm{C}, 1.0 \mathrm{~mL} \mathrm{~min}^{-1}$ ) versus polystyrene standards on a Hewlett-Packard series 1100 HPLC equipped with Polymer Laboratories 5- $\mu \mathrm{m}$ mixed-C columns and connected to UV-vis and refractive index (Viscotek LR 40) detectors. Data were processed with the OmniSEC software package (version 4.2, Viscotek Corp). A 0.58 correction factor was applied to determine molecular weights of $\mathbf{1}$ as previously described. ${ }^{43,51} \mathrm{UV}$-vis spectra were recorded on a Hewlett-Packard 8453 diode array spectrophotometer. ${ }^{1} \mathrm{H}$ NMR $(300 \mathrm{MHz})$ spectra were recorded on a Unitylnova 300/51 instrument and referenced to the signal for residual protio chloroform at $7.26 \mathrm{ppm}$. Photographs of luminescent solutions were taken in the dark using a Canon PowerShot SD600 Digital Elph camera with the automatic setting (no flash).

Fluorescence and Phosphorescence Measurements. Steady-state fluorescence and phosphorescence emission spectra were recorded on a Horiba Fluorolog-3 Model FL3-22 spectrofluorimeter (double-grating excitation and double-grating emission monochromators). Prior to recording phosphorescence emission spectra, the aqueous nanoparticle solution $(\sim 3 \mathrm{~mL})$ was purged with $\mathrm{N}_{2}$ for 20 min in a quartz fluorimeter cell equipped with a septum screw top. RTP spectra were recorded under the same conditions as phosphorescence spectra except that a pulsed xenon lamp ( $\lambda_{\text {ex }}=369 \mathrm{~nm}$; duration $<1 \mathrm{~ms}$ ) was used and spectra were collected with a $1 \mathrm{~ms}$ delay after excitation. Timecorrelated single-photon counting fluorescence lifetime measurements were carried out with a NanoLED-370 (369 nm) excitation source and a DataStation Hub as the single-photon counting controller. Phosphorescence lifetimes were measured with a 500 ns multichannel scalar card excited with a pulsed xe- non lamp ( $\lambda_{\mathrm{ex}}=369 \mathrm{~nm}$; duration $<1 \mathrm{~ms}$ ). Lifetime data were analyzed with DataStation v2.4 software from Horiba Jobin Yvon.

Nanoparticle Fabrication. A representative preparation is as follows: an equimolar mixture of $1\left(20 \mathrm{mg}, M_{\mathrm{n}}=5700 \mathrm{Da}\right)$ and 2 (24 mg, $M_{\mathrm{n}}=6600 \mathrm{Da}$ ) was dissolved in either 10:1 (v/v) DMF/ THF or DMF only ( $4.4 \mathrm{~mL})$. In the case of DMF, the solution was briefly heated and sonicated to ensure complete dissolution. The resulting solution was then cooled to room temperature and added dropwise via syringe to $\mathrm{H}_{2} \mathrm{O}(44 \mathrm{~mL})$ at a rate of $0.5 \mathrm{~mL}$ $\min ^{-1}$. During addition of the solution, the $\mathrm{H}_{2} \mathrm{O}$ phase was stirred at a fast rate, and the resulting homogeneous suspension was stirred an additional $30 \mathrm{~min}$. The suspension was then dialyzed using dialysis tubing (SpectraPor, 12-14 kDa MWCO, Fisher Scientific) against distilled $\mathrm{H}_{2} \mathrm{O}$ under slow stirring conditions according to the literature for complete removal of DMF or DMF/ THF. Following dialysis the aqueous nanoparticle suspension was passed through filter paper (Whatman, Qualitative grade 2) and stored in vials. The suspension was characterized as is or freezedried for further characterization.

Nanoparticle Size Determination. Nanoparticle sizes were determined by dynamic light scattering (DLS) $\left(90^{\circ}\right.$ angle) on a Photocor Complex (Photocor Instruments Inc., USA) equipped with a $\mathrm{He}-\mathrm{Ne}$ laser (Coherent, USA, Model 31-2082, $632.8 \mathrm{~nm}, 10 \mathrm{~mW}$ ). Size and polydispersity analyses were performed using DynaLS software (Alango, Israel).

0xygen Sensitivity Calibration. For oxygen sensitivity and intensity-based Stern-Volmer plot measurements, gas mixtures of $\mathrm{O}_{2}$ and $\mathrm{N}_{2}$ of various concentrations were prepared by using two Cole-Parmer 65-mm flow meters. For each concentration, the premixed gas was first evenly dispensed into a distilledwater chamber before being passed through a 1-cm-path quartz fluorometer cell equipped with a septum screw top containing 1 $\mathrm{mL}$ of the aqueous nanoparticle suspension.

Window Chambers for Rodent Studies. A dorsal skin fold window chamber was implanted into a nude mouse, and a 4T1 murine mammary tumor was inoculated using established procedures. ${ }^{52}$ The tumor was allowed to grow for $10 \mathrm{~d}$ prior to imaging.

Confocal Microscopy Imaging Setup. Fluorescence and transmission images were acquired using a Leica SP5 confocal microscope. This system is equipped with an acousto-optical tunable filter for both the excitation and emission, enabling multispectral imaging with tunable wavelength ranges, as well as multiple 
PMT detectors for simultaneous acquisition of multiple channels. A $10 \times$ dry objective was used along with a $405 \mathrm{~nm}$ laser diode as the excitation source. Three separate collection channels were used: (1) band-pass emission from 420 to $495 \mathrm{~nm}$ (BNP fluorescence), (2) band-pass emission from 496 to $623 \mathrm{~nm}$, (BNP phosphorescence) and (3) a transmission image. The scanning speed was set to $10 \mathrm{~Hz}$.

Confocal Imaging Experiments. Aqueous BNPs $(100 \mu \mathrm{L})$ were injected via the tail vein. Images were acquired prior to injection and semicontinuously monitored for $\sim 80$ min postinjection.

Confocal Imaging Data Analysis. Vessels were segmented using a regional intensity threshold by selecting pixels with a lower than mean value based on a 15 pixel radius moving window. Morphological opening using linear structural elements at angle increments of $15^{\circ}$ was then done to isolate linear segments. The accumulation could then be tracked as a function of time for the vascular and surrounding tissue BNP accumulation.

Acknowledgment. We thank the following people for experimental assistance and use of facilities: Prof. D. L. Green and M. McEwan (DLS). This work was supported by the National Science Foundation (C.L.F.: CHE 0718879), the UVA NanoSTAR Institute, the UVA Cancer Center through the James and Rebecca Craig Foundation and the $\mathrm{NCl}$ Cancer Center Support Grant (C.L.F.: P30 CA44579), the Department of Defense (G.M.P.: Postdoctoral Fellowship W81XWH-07-1-0355), and the National Institutes of Health (M.W.D.: R01CA40355). We also gratefully acknowledge Purac for their generous gift of D-lactide.

Supporting Information Available: Oxygen calibration plot over full range of $\mathrm{O}_{2}$ concentrations, GPC UV signal traces, and plots of RTP decrease/fluorescence shift over time. This materia is available free of charge via the Internet at http://pubs.acs.org.

\section{REFERENCES AND NOTES}

1. Riess, G. Micellation of Block Copolymers. Prog. Polym. Sci. 2003, 28, 1107-1170.

2. Discher, D. E.; Ahmed, F. Polymersomes. Annu. Rev. Biomed. Eng. 2006, 8, 323-341.

3. Allen, C.; Maysinger, D.; Eisenberg, A. Nano-Engineering Block Copolymer Aggregates for Drug Delivery. Colloids Surf. B 1999, 16, 3-27.

4. Burt, H. M.; Zhang, X.; Toleikis, P.; Embree, L.; Hunter, W. L. Development of Copolymers of Poly(D,L-lactide) and Methoxypolyethylene Glycol as Micellar Carriers of Paclitaxel. Colloids Surf. B 1999, 16, 161-171.

5. Chen, L.; Xie, Z.; Hu, J.; Chen, X.; Jing, X. Enantiomeric PLAPEG Block Copolymers and their Stereocomplex Micells used as Rifampin Delivery. J. Nanopart. Res. 2007, 9, 777785.

6. Kim, S. C.; Kim, D. W.; Shim, Y. H.; Bang, J. S.; Oh, H. S.; Kim, S. W.; Seo, M. H. In Vivo Evaluation of Polymeric Micellar Paclitaxel Formulation: Toxicity and Efficacy. J. Controlled Release 2001, 72, 191-202.

7. Maeda, H.; Wu, J.; Sawa, T.; Matsumura, Y.; Hori, K. Tumor Vascular Permeability and the EPR Effect in Macromolecular Therapeutics: A Review. J. Controlled Release 2000, 65, 271-284.

8. Dreher, M. R.; Liu, W.; Michelich, C. R.; Dewhirst, M. W.; Yuan, F.; Chilkoti, A. Tumor Vascular Permeability, Accumulation, and Penetration of Macromolecular Drug Carriers. J. Natl. Cancer Inst. 2006, 98, 335-344.

9. Salem, A. K.; Cannizzaro, S. M.; Davies, M. C.; Tendler, S. J. B.; Roberts, C. J.; Williams, P. M.; Shakesheff, K. M. Synthesis and Characterisation of a Degradable Poly(lactic acid)-Poly(ethylene glycol) Copolymer with Biotinylated End Groups. Biomacromolecules 2001, 2, 575-580.

10. Pulkkinen, M.; Pikkarainen, J.; Wirth, T.; Tarvainen, T.; Haapa-aho, V.; Korhonen, H.; Seppälä, J.; Järvinen, K. ThreeStep Tumor Targeting of Paclitaxel Using Biotinylated PLA-PEG Nanoparticles and Avidin-Biotin Technology: Formulation Development and In Vitro Anticancer Activity. Eur. J. Pharm. Biopharm. 2008, 70, 66-74.

11. Patil, Y. B.; Toti, U. S.; Khdair, A.; Ma, L.; Panyam, J. SingleStep Surface Functionalization of Polymeric Nanoparticles for Targeted Drug Delivery. Biomaterials 2009, 30, 859-866.

12. Olivier, J.-C.; Huertas, R.; Lee, H. J.; Calon, F.; Pardride, W. M. Synthesis of Pegylated Immunonanoparticles. Pharm. Res. 2002, 19, 1137-1143

13. Hagan, S. A.; Coombes, A. G. A.; Garnett, M. C.; Dunn, S. E.; Davies, M. C.; Illum, L.; Davis, S. S.; Harding, S. E.; Purkiss, S.; Gellert, P. R. Polylactide-Poly(ethylene glycol) Copolymers as Drug Delivery Systems. 1. Characterization of Water Dispersible Micelle-Forming Systems. Langmuir 1996, 12 2153-2161.

14. Kim, S. Y.; Shin, I. G.; Lee, Y. M. Preparation and Characterization of Biodegradable Nanospheres Composed of Methoxy Poly(ethylene glycol) and DLLactide Block Copolymer as Novel Drug Carriers. J. Controlled Release 1998, 56, 197-208.

15. Avgoustakis, K. Pegylated Poly(lactide) and Poly(lactideco-glycolide) Nanoparticles: Preparation, Properties and Possible Applications in Drug Delivery. Curr. Drug Delivery 2004, 1, 321-333.

16. Yang, L.; Zhao, Z.; Wei, J.; Ghzaoui, A. E.; Li, S. Micelles Formed by Self-Assembling of Polylactide/Poly(ethylene glycol) Block Copolymers in Aqeuous Solutions. J. Colloid Interface Sci. 2007, 314, 470-477.

17. Ma, L. L.; Jie, P.; Venkatraman, S. S. Block Copolymer "Stealth" Nanoparticles for Chemotherapy: Interactions with Blood Cells In Vitro. Adv. Funct. Mater. 2008, 18, 716725.

18. Mosqueira, V. C. F.; Legrand, P.; Morgat, J.-L.; Vert, M.; Mysiakine, E.; Gref, R.; Devissaguet, J.-P.; Barratt, G. Biodistribution of Long-Circulating PEG-Grafted Nanocapsules in Mice: Effects of PEG Chain Length and Density. Pharm. Res. 2001, 18, 1411-1419.

19. Kim, T.-Y.; Kim, D.-W.; Chung, J.-Y.; Shin, S. G.; Kim, S.-C.; Heo, D. S.; Kim, N. K.; Bang, Y.-J. Phase I and Pharmacokinetics Study of Genexol-PM, a CremophorFree, Polymeric Micelle-Formualted Paclitaxel, in Patients with Advanced Malignancies. Clin. Cancer Res. 2004, 10, 3708-3716.

20. Jie, P.; Venkatraman, S. S.; Min, F.; Freddy, B. Y. C.; Huat, G. L. Micelle-Like Nanoparticles of Star-Branched PEO-PLA Copolymers as Chemotherapeutic Carrier. J. Controlled Release 2005, 110, 20-33.

21. Kang, N.; Perron, M.-E.; Prud'homme, R. E.; Zhang, Y.; Gaucher, G.; Leroux, J.-C. Stereocomplex Block Copolymer Micelles: Core-Shell Nanostructures with Enhanced Stability. Nano Lett. 2005, 5, 315-319.

22. Hu, J.; Han, Y.; Zhuang, X.; Chen, X.; Li, Y.; Jing, X. SelfAssembly of a Polymer Pair through Poly(lactide) Stereocomplexation. Nanotechnology 2007, 18, 185607.

23. Kim, S. H.; Tan, J. P. K.; Nederberg, F.; Fukushima, K.; Yang, Y. Y.; Waymouth, R. M.; Hedrick, J. L. Mixed Micelle Formation through Stereocomplexation between Enantiomeric Poly(lactide) Block Copolymers. Macromolecules 2009, 42, 25-29.

24. Tan, J. P. K.; Kim, S. H.; Nederberg, F.; Appel, E. A.; Waymouth, R. M.; Zhang, Y.; Hedrick, J. L.; Yang, Y. Y. Hierarchical Supermolecular Structures for Sustained Drug Release. Small 2009, 5, 1504-1507.

25. Loudet, A.; Burgess, K. BODIPY Dyes and Their Derivatives: Syntheses and Spectroscopic Properties. Chem. Rev. 2007, 107, 4891-4932.

26. Chow, Y. L.; Johansson, C. I.; Zhang, Y.-H.; Gautron, R.; Yang, L.; Rassat, A.; Yang, S.-Z. Spectroscopic and Electrochemical Properties of 1,3-Diketonatoboron Derivatives. J. Phys. Org. Chem. 1996, 9, 7-16.

27. Carraway, E. R.; Demas, J. N.; DeGraff, B. A.; Bacon, J. R. Photophysics and Photochemistry of Oxygen Sensors Based on Luminescent Transition-Metal Complexes. Anal. Chem. 2002, 63, 337-342.

28. Guice, K. B.; Caldorera, M. E.; McShane, M. J. Nanoscale Internally Referenced Oxygen Sensors Produced from SelfAssembled Nanofilms on Fluorescent Nanoparticles. J. Biomed. Opt. 2005, 10, 064031 
29. Sun, H.; Scharff-Poulsen, A. M.; Gu, H.; Almdal, K. Synthesis and Characterization of Ratiometric, $\mathrm{pH}$ Sensing Nanoparticles with Covalently Attached Fluorescent Dyes. Chem. Mater. 2006, 18, 3381-3384.

30. Somers, R. C.; Bawendi, M. G.; Nocera, D. G. CdSe Nanocrystal Based Chem-/Bio- Sensors. Chem. Soc. Rev. 2007, 36, 579-591.

31. Snee, P. T.; Somers, R. C.; Nair, G.; Zimmer, J. P.; Bawendi, M. G.; Nocera, D. G. A Ratiometric CdSe/ZnS Nanocrystal pH Sensor. J. Am. Chem. Soc. 2006, 128, 13320-13321.

32. McLaurin, E. J.; Greytak, A. B.; Bawendi, M. G.; Nocera, D. G. Two-Photon Absorbing Nanocrystal Sensors for Ratiometric Detection of Oxygen. J. Am. Chem. Soc. 2009, 131, 12994-13001.

33. Wu, C.; Bull, B.; Christensen, K.; McNeill, J. Ratiometric Single-Nanoparticle Oxygen Sensors for Biological Imaging. Angew. Chem., Int. Ed. 2009, 48, 2741-2745.

34. Koo Lee, Y.-E.; Smith, R.; Kopelman, R. Nanoparticle PEBBLE Sensors in Live Cells and In Vivo. Ann. Rev. Anal. Chem. 2009, 2, 57-76.

35. Xu, H.; Aylott, J. W.; Kopelman, R.; Miller, T. J.; Philbert, M. A. A Real-Time Ratiometric Method for the Determination of Molecular Oxygen Inside Living Cells Using Sol-Gel-Based Spherical Optical Nanosensors with Applications to Rat C6 Glioma. Anal. Chem. 2001, 73, 4124-4133.

36. Koo, Y.-E. L.; Cao, Y.; Kopelman, R.; Koo, S. M.; Brasuel, M.; Philbert, M. A. Real-Time Measurements of Dissolved Oxygen Inside Live Cells by Organically Modified Silicate Fluorescent Nanosensors. Anal. Chem. 2004, 76, 2498-2505.

37. Lebedev, A. Y.; Cheprakov, A. V.; Sakadzic, S.; Boas, D. A.; Wilson, D. F.; Vinogradov, S. A. Dendritic Phosphorescent Probes for Oxygen Imaging in Biological Systems. ACS Appl. Mater. Interfaces 2009, 1, 1292-1304.

38. Brinas, R. P.; Troxler, T.; Hochstrasser, R. M.; Vinogradov, S. A. Phosphorescent Oxygen Sensor with Dendritic Protection and Two-Photon Absorbing Antenna. J. Am. Chem. Soc. 2005, 127, 11851-11862.

39. Zhang, G.; Chen, J.; Payne, S. J.; Kooi, S. E.; Demas, J. N.; Fraser, C. L. Multi-Emissive Difluoroboron Dibenzoylmethane Polylactide Exhibiting Intense Fluorescence and Oxygen-Sensitive Room-Temperature Phosphorescence. J. Am. Chem. Soc. 2007, 129, 8942-8943.

40. Zhang, G.; Kooi, S. E.; Demas, J. N.; Fraser, C. L. Emission Color Tuning with Polymer Molecular Weight for Difluroboron Dibenzoylmethane-Polylactide. Adv. Mater. 2008, 20, 2099-2104.

41. Pfister, A.; Zhang, G.; Zareno, J.; Horwitz, A. F.; Fraser, C. L. Boron Polylactide Nanoparticles Exhibiting Fluorescence and Phosphorescence in Aqueous Medium. ACS Nano 2008, 2, 1252-1258.

42. Contreras, J.; Xie, J.; Chen, Y. J.; Pei, H.; Zhang, G.; Fraser, C. L.; Hamm-Alvarez, S. F. Intracellular Uptake and Trafficking of Difluoroboron Dibenzoylmethane-Poly(lactic acid) Nanoparticles in HeLa Cell. ACS Nano 2010, 4, 2735-2747.

43. Zhang, G.; Palmer, G. M.; Dewhirst, M. W.; Fraser, C. L. A Dual-Emissive-Materials Design Concept Enables Tumour Hypoxia Imaging. Nat. Mater. 2009, 8, 747-751.

44. Fessi, H.; Puisieux, F.; Devissaguet, J. P.; Ammoury, N.; Benita, S. Nanocapsule Formation by Interfacial Polymer Deposition Following Solvent Displacement. Int. J. Pharm. 1989, 55, R1-R4.

45. Sánchez-Barragán, I.; Costa-Fernández, J. M.; Sanz-Medel, A.; Valledor, M.; Campo, J. C. Room-Temperature Phosphorescence (RTP) for Optical Sensing. Trends Anal. Chem. 2006, 25, 958-967.

46. Hamburger, R.; Azaz, E.; Donbrow, M. Autoxidation of Polyoxyethylenic Non-lonic Surfactants and of Polyethylene Glycols. Pharm. Acta Helv. 1975, 50, 10-17.

47. Chow, Y. L.; Wang, S.-S. The Photocycloaddition of Dibenzoylmethanatoboron Difluoride (DBMBF2) with Diconjugated Enones and En-esters. Can. J. Chem. 1993, $71,846-854$
48. Chow, Y. L.; Ouyang, X. The Photoaddition of 1,3Diketonatoboron Difluorides with Benzene Derivatives. Can. J. Chem. 1991, 69, 423-431.

49. De Jaeghere, F.; Allémann, E.; Leroux, J.-C.; Stevels, W.; Feijen, J.; Doelker, E.; Gurny, R. Formulation and Lyoprotection of Poly(Lactic Acid-Co-Ethylene Oxide) Nanoparticles: Influence on Physical Stability and In Vitro Cell Uptake. Pharm. Res. 1999, 16, 859-866.

50. De Jaeghere, F.; Allemann, E.; Feijen, J.; Kissel, T.; Doelker, E.; Gurny, R. Freeze-Drying and Lyopreservation of Diblock and Triblock Poly(Lactic Acid)-Poly(Ethylene Oxide) (PLAPEO) Copolymer Nanoparticles. Pharm. Dev. Technol. 2000, 5, 473-483.

51. Bender, J. L.; Corbin, P. S.; Fraser, C. L.; Metcalf, D. H.; Richardson, F. S.; Thomas, E. L.; Urbas, A. M. Site-lsolated Luminescent Europium Complexes with Polyester Macroligands: Metal-Centered Heteroarm Stars and Nanoscale Assemblies with Labile Block Junctions. J. Am. Chem. Soc. 2002, 124, 8526-8527.

52. Huang, Q.; Shan, S.; Braun, R. D.; Lanzen, J.; Anyrhambatla, G.; Kong, G.; Borelli, M.; Corry, P.; Dewhirst, M. W.; Li, C.Y. Noninvasive Visualization of Tumors in Rodent Dorsal Skin Window Chambers. Nat. Biotechnol. 1999, 17, 1033-1035. 